\title{
Spinal Cord Compression in Breast Cancer
}

Kriouile $\mathrm{K}^{1 *}$, Afalah $\mathrm{H}^{1}$, Jayi. $\mathrm{S}^{1}$, Fdili Alaoui. $\mathrm{FZ}^{1}$, Chaara. $\mathrm{H}^{1}$, Melhouf My A ${ }^{1}$, H. Ouahbi ${ }^{2}$, N. Mellas ${ }^{2}$

${ }^{1}$ Department of Obstetric Gynecology, Hassan II University Hospital, Fez, Morocco

${ }^{2}$ Department of Medical Oncology, Hassan II University Hospital, Fez, Morocco

DOI: $10.36347 /$ simcr.2020.v08i02.041

| Received: 10.02.2020 | Accepted: 19.02.2020 | Published: 28.02.2020

*Corresponding author: Kriouile $\mathrm{K}$

Abstract

Original Research Article

Breast cancer is the most common malignancy and the first leading cause of death from cancer in Moroccan women. Breast cancer most commonly metastasizes to the bone and has a particular affinity with the spine, accounting for $2 / 3$ of osseous metastases discovered. With significant improvements in cancer therapies, the number of patients at risk for symptomatic spinal metastases is likely to increase. Patients may suffer from intractable pain and neurological dysfunction, negatively influencing their quality of life. Timely diagnosis of patients is crucial and has been aided by several breakthrough advances in imaging techniques which aid in detection, staging, and follow-up of bone metastases. Breast metastases are usually responsive to hormonal therapy and pharmacologic interventions, but skeletal metastases often require surgical intervention. The treatments are palliative but goals include the preserving or restoring neurologic function, ensuring spinal stability, and relieving pain. In this case report, the clinical presentation, diagnosis, patient selection, and management of breast cancer metastatic to the spine are discussed.

Keywords: Breast cancer; Spine; Metastasis; Decompression.

Copyright @ 2020: This is an open-access article distributed under the terms of the Creative Commons Attribution license which permits unrestricted use, distribution, and reproduction in any medium for non-commercial use (NonCommercial, or CC-BY-NC) provided the original author and source are credited.

\section{INTRODUCTION}

Metastatic spinal cord compression is an oncological emergency and can lead to irreversible functional sequelae, leading to an alteration in the quality of life of the cancer patient whose early diagnosis and appropriate management can improve the prognosis. The aim of this work is to evaluate the management of spinal cord compression of metastatic breast cancer, which is a frequent reason for hospitalization in our context.

\section{Material ANd Methods}

This is a retrospective study of 34 follow-up patients for a metastatic breast tumor hospitalized betwin the Department of Obstetric Gynecology and the Department of Medical Oncology University Hospital Hassan II of Fez during the period between January 2016 and December 2019 admitted for a Metastatic medullary compression syndrome.

\section{RESULTS}

The mean age of the patients was $49 \pm 7.73$, the molecular profile was dominated by the luminal type B HER 2 negative in 50\%, luminal A in $33 \%$ and the triple negative in $22 \%$. The median follow-up time after symptom onset was 10 days, revealing clinical signs were dominated by Spinal Syndrome in $11.1 \%$ (n $=2)$, and lesional syndrome in $33.3 \%(n=6)$ and underlesional syndrome in $55.6 \%(\mathrm{n}=10)$ of the cases, with flaccid paraplegia in $27 \%, 39 \%$ of the patients benefited from spinal MRI, medullary compression was expressed by infiltration of Conjugation hole in $28 \%$ of cases, and epidural space in $89 \%$. The site of the lesion was predominantly at the level of the dorsal spine in $78 \%$ of the cases. Symptomatic treatment was administered in all patients (PPI, analgesic ...), corticotherapy was administered in $100 \%$ of cases, and radiotherapy was administered for decompressive purpose in $79 \%$ of the cases. $11 \%$ of patients undergoing decompressive surgery followed by consolidation radiotherapy, chemotherapy were administered after completion of radiotherapy in all patients. Evolution was marked by an improvement in symptomatology with neurological recovery in $72 \%$ of cases, and persistence of deficit in $27 \%$ of cases. The complications of decubitus were observed in a single patient who presented both deep venous thromboses of the lower limbs with sacral wounds.

\section{Discussion}

Multiple malignancies such as breast, prostate, kidney, and lung show a remarkable affinity to metastasize to bone [1]. Metastatic lesions can spread to 
the bone via several mechanisms, but the method of dissemination most likely responsible for breast cancer involves hematogenous seeding via venous routes [2]. Spread may be accomplished through the Batson plexus, a network of veins that connects the vertebral veins with other beds of venous drainage. This importantly includes the azygos vein, which receives blood draining from the breast via the intercostal veins. The venous plexus of Batson lacks valves to control the flow of blood, so changes in pressure within the body can lead to variable flow through the plexus, allowing retrograde or antegrade seeding of tumor cells [3]. The exact mechanism of metastatic seeding of the bone is unclear. There exists a prominent disparity between the abundance of circulating tumor cells and the relative rarity of metastatic seeding, suggesting a complex environmental barrier to metastasis [4]. Nevertheless, when metastatic events occur in the spine they most commonly occur within the vertebral body with or without extension into the posterior elements [3].

Pain is the most common symptom and is the presenting complaint in nearly $90 \%$ of patients with spinal metastases from breast cancer [6]. Pain symptoms vary in intensity but may be vague and nonspecific, and patients with metastatic spinal cord compression have been found to have a delay in diagnosis of about 2 mo from first presenting to a physician to the time of diagnosis [7]. As the neurological status of the patient at time of diagnosis correlates strongly to the patient's prognosis, a diagnosis before the onset of neurological compromise is essential [5,8]. Accordingly, any patient with a known history of malignancy who presents with newonset back or neck pain should be promptly and thoroughly evaluated with a high suspicion for metastatic disease involving the spine. Common degenerative disorders less commonly affect the thoracic spine than the cervical or lumbar spine, hence pain in the thoracic spine warrants a high clinical suspicion for metastatic disease [5]. Likewise, patients with persistent nonmechanical pain should have a low threshold for evaluation of a neoplastic etiology [9].
The available imaging modalities to evaluate for suspected breast cancer metastatic to the spine include plain radiographs, skeletal scintigraphy (SS) (bone scan), computed tomography (CT), magnetic resonance imaging (MRI), positron emission tomography CT (PET-CT), and single photon emission CT (SPECT). These modalities carry varying sensitivities, degrees of information, and costs in evaluating for spinal metastases. Given the ability of metastatic breast cancer to present as osteoblastic, osteolytic, or mixed lesions in the bone, each imaging technique may play a valuable role in the evaluation of the at-risk patient depending on the clinical situation [10].

The management of patients with metastatic breast cancer to the spine is complex and frequently requires a multidisciplinary approach, involving numerous medical specialties (oncology, radiation oncology, pain management, rehabilitation medicine), surgery subspecialties (neurosurgery, orthopedics, surgical oncology), as well as radiologists and interventional radiologists. Advances in the past few decades have improved the treatment of both systemic disease as well as localized tumor burden to the spine. Cytotoxic agents remain the mainstay of treatment of patients with breast cancer. Hormone therapies such as selective estrogen receptor modulators and aromatase inhibitors have been shown to be effective against breast cancer, and human epidermal growth factor receptor 2 (HER2) targeting agents have also been effective in treating metastatic patients[11,12]. Moreover, bisphosphonates given with vitamin $\mathrm{D}$ and calcium, which inhibit tumor-related osteoclast activity, and corticosteroids, which may have oncolytic effects on breast cancer and decrease peritumor edema, give physicians an ever-growing array of tools to combat this disease. However, the treatment for symptomatic spinal metastases remains palliative and is not intended to prolong survival. The goals of treatment include restoration of and preservation of neurological function, maintaining spinal stability, and pain relief in an effort to achieve a better quality of life. A proposed algorithm for the treatment of patients with symptomatic spinal metastasis from breast cancer is shown in Figure 1. 


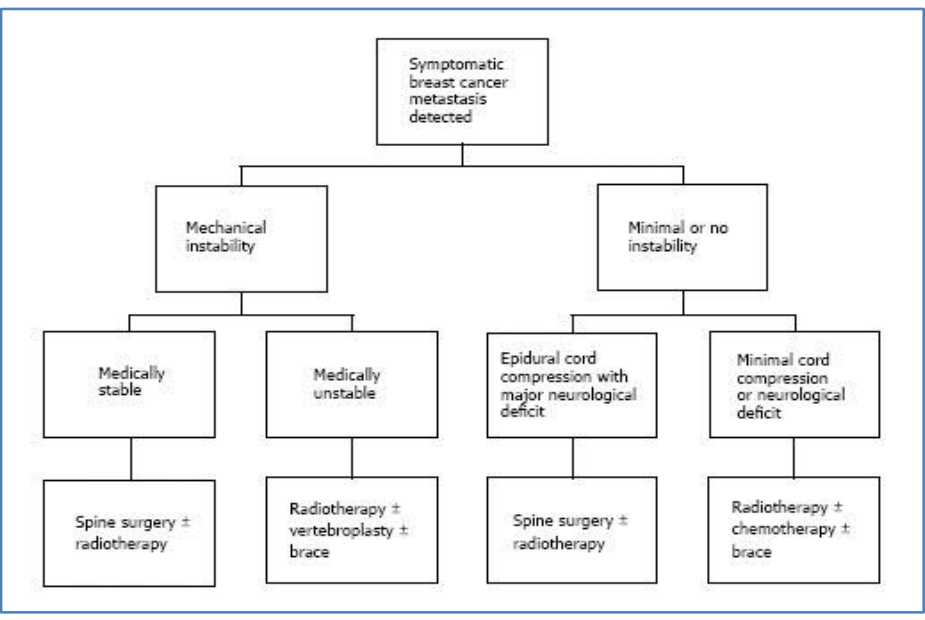

Fig-1: Proposed algorithm for treatment of patients with symptomatic spinal metastases from breast cancer

The prevalence of patients with a history of breast cancer is increasing as the rate of survival improves, highlighting the large population of patients at risk for symptomatic spinal metastases. The management of patients with metastatic breast cancer to the spine is often complex and requires a multidisciplinary approach. Precise diagnosis with history, physical, and imaging are imperative to initiate the appropriate treatment in a timely manner. The treatment for symptomatic spinal metastases remains palliative and is not intended to prolong survival. Surgical advances in the last few decades have allowed improved spinal cord decompression and tumor resection and continue to evolve. The goals of treatment include restoration of and preservation of neurological function, maintaining spinal stability, and pain relief in an effort to achieve a better quality of life. Further research should focus on pathology-specific whenever possible, given its implications for treatment selection and prognosis [13].

\section{CONCLUSION}

Spinal cord compression secondary to metastatic breast cancer is frequent in oncology; it presents a diagnostic and therapeutic urgency which may involve the functional and vital prognosis of the patient, hence the value of an early diagnosis and adequate medical care.

\section{REFFERNCE}

1. Lu X, Kang Y. Organotropism of breast cancer metastasis.J Mammary Gland Biol Neoplasia. 2007; 12: 153-162 [PMID:17566854]

2. Maccauro G, Spinelli MS, Mauro S, Perisano C, Graci C, Rosa MA. Physiopathology of spine metastasis. Int J Surg Oncol. 2011;2011: 107969

3. Sciubba DM, Petteys RJ, Dekutoski MB, Fisher CG, Fehlings MG, Ondra SL, Rhines LD, Gokaslan ZL. Diagnosis and management of metastatic spine disease. A review. J Neurosurg Spine. 2010; 13: 94-108
4. Gupta GP, Massagué J. Cancer metastasis: building a framework. Cell. 2006; 127: 679-695

5. Quraishi NA, Gokaslan ZL, Boriani S. The surgical management of metastatic epidural compression of the spinal cord. J Bone Joint Surg Br. 2010; 92: 1054-1060

6. Landreneau FE, Landreneau RJ, Keenan RJ, Ferson PF. Diagnosis and management of spinal metastases from breast cancer. J Neurooncol. 1995; 23: $121-134$

7. Levack P, Graham J, Collie D, Grant R, Kidd J, Kunkler I, Gibson A, Hurman D, McMillan N, Rampling R, Slider L, Statham P, Summers D. Don't wait for a sensory level--listen to the symptoms: a prospective audit of the delays in diagnosis of malignant cord compression. Clin Oncol (R Coll Radiol). 2002; 14: 472-480.

8. Zadnik PL, Hwang L, Ju DG, Groves ML, Sui J, Yurter A, Witham TF, Bydon A, Wolinsky JP, Gokaslan ZL, Sciubba DM. Prolonged survival following aggressive treatment for metastatic breast cancer in the spine. Clin Exp Metastasis. 2014; 31: 47-55.

9. Molina CA, Gokaslan ZL, Sciubba DM. Diagnosis and management of metastatic cervical spine tumors. Orthop Clin North Am. 2012; 43: 75-87.

10. Schirrmeister H. Detection of bone metastases in breast cancer by positron emission tomography. Radiol Clin North Am. 2007; 45: 669-676.

11. Jelovac D, Emens LA. HER2-directed therapy for metastatic breast cancer. Oncology (Williston Park) 2013; 27: 166-175

12. Morandi P, Rouzier R, Altundag K, Buzdar AU, Theriault RL, Hortobagyi G. The role of aromatase inhibitors in the adjuvant treatment of breast carcinoma: the M. D. Anderson Cancer Center evidence-based approach. Cancer. 2004; 101: 1482-1489

13. Derek G Ju, Alp Yurter, Ziya L Gokaslan, Daniel M Sciubba; Diagnosis and surgical management of breast cancer metastatic to the spine; World J Clin Oncol. 2014 August 10; 5(3): 263-271. 